\title{
Game-Theoretic Analysis of a Web Services Collaborative Mechanism
}

\author{
Babak Khosravifar ${ }^{1}$, Jamal Bentahar ${ }^{1}$, Kathleen Clacens ${ }^{2}$, \\ Christophe Goffart ${ }^{2}$, and Philippe Thiran ${ }^{2}$ \\ 1 Concordia University, Montreal, Canada \\ ${ }^{2}$ University of Namur, Namur, Belgium \\ b_khosr@encs.concordia.ca, bentaharaciise.concordia.ca, \\ $\{$ k.clacens, darkyunsung\}@gmail.com, pthiran@fundp.ac.be
}

\begin{abstract}
Web services are business applications having the capability to cooperate within groups to increase the efficiency of serving customers. There have been a number of proposed frameworks aggregating web services for the purpose of enhancing their capabilities with respect to providing the required service. However, the grouping procedure has got less attention. In this paper, we discuss the mechanism web services can use to join existing groups of web services (known as communities). Moreover, we analyze the scenarios where the community is filled up with web services that lied about their capabilities before joining. The objective is to provide and maintain a truthful environment where involving components act truthfully.
\end{abstract}

Keywords: Web services, Reputation, Agents.

\section{Introduction}

During recent years, web services have obtained a strong attention as they represent distributed cooperation in business and IT networks. Web services hold predefined capabilities that let them realize their goals by engaging in interactions with one another. They are loosely-coupled business applications and willing to cooperate in distributed settings for the sake of efficiency. To this end, there have been efforts made in collaboration between web services [4]. The objective of this collaboration is to increase web services' capabilities. However, web services also need to be located in environments where requests are received from users in continuous manner, which requires a high reputation. Furthermore, there are many aspects that should be considered when analyzing web services working within groups (generally called communities). In this paper, we mainly concentrate on the joining aspect of web services to existing communities by focusing on their rational behaviors. The concept of community of web services together with its relative details are explained in Section 2.4

In this paper, we present a game-theoretical model analyzing the communities of web services from the perspective of hosting different web services. A game is defined between the master web service acting as the representative of the community and agents acting as information providers within a group called information service group. 
Agents in this group, called information service agents, provide the necessary information regarding the web service that is attempting to join a community. The involved information service agents can either lie or tell the truth about the requested information. A payment mechanism that provides diverse ranges of payoffs to the information service agents according to their chosen strategies is discussed and analyzed. Overall contribution of the paper is summarized in game-theoretic analysis investigating the stabilized situation where information service agents provide their actual information and act truthfully.

\section{Related Preliminaries}

\subsection{Web Services}

The main motivation behind the use of web services technology is the development of loosely-coupled, cross-enterprize business processes. This means web services can be used without worrying about how they are implemented or where in the world they are hosted. As in [45] and [8], we abstract web services as rational intelligent agents, which are benefit maximizers. In our framework, the goal of these agents is to receive user requests and satisfy them the best they can.

\subsection{Information Service Agents}

Information service agents provide a ranking of web services. They know all the active web services and depending on their personal behavior and incentives, they could provide accurate or non-accurate information about these web services.

\subsection{Reputation}

As for non-internet based services, for example buying a plane ticket from a travel agency, it is possible that several web services have the same functionalities. We therefore need to differentiate those different, but functionally similar web services. We will use the concept of reputation that strongly depends on the quality of service (QoS). The Quality represents the capability of a web service to handle the users' requests in a timely fashion. In order to use this concept of reputation, we use a system architecture having the following elements: (1) some registries containing entries that describe individual web services; and (2) a reputation mechanism [8]. The registries can be implemented using the UDDI protocol, which defines a standard method for publishing and discovering web services [3].

\subsection{Community of Web Services}

A web service alone can easily be overloaded by an intense flow of user's requests. It will lead to a poor efficiency and therefore a drop in the users' satisfaction and reputation [6]. As argued in [4] and [6], a solution is to group different web services 
in a community. By aggregating the total number of requests that each single web service can handle, and redistributing them among all the members, a community will be granted a better availability and hence better efficiency.

In a community, we distinguish between two types of web services: a unique community master and two or more community slaves. The master takes the responsibility of leading the community. He must attract new web services in the community by using rewards, convince web services to stay in the community, and select the web service that will respond to a user's request. When a user sends a request to a community, the master has to nominate a slave from his community that can handle that request.

\section{The Model}

\subsection{The Modelled Structure}

In general, when a customer asks an information service agent, there are two possible strategies: lying or telling the truth. The utility $u_{k}(x)$ of an information service agent $k$ choosing the strategy $x$ is a function having 3 incentive components where the agent obtains rewards or penalties according to the chosen strategy. It is defined as follows:

$$
\begin{gathered}
u_{k}(x)=f_{k}(x)+g_{k}(x)+c . h_{k}(x) \text { where } \\
f_{k}(x)>0, f_{k}(x)<\left|g_{k}(x)\right| \text { and } f_{k}(x)+\left|g_{k}(x)\right|<\left|h_{k}(x)\right|
\end{gathered}
$$

The first component $f_{k}(x)$ is a positive reward that a customer gives to the information service agent $k$ who is willing to provide the asked information. The second component incentive $g_{k}(x)$ corresponds to a value that will be granted to the information service agent depending on the similarity between the information she gives and the average information revealed by the other information service agents. This second value can be negative if the distance is high and therefore acts as a punishment preventing the information service agent to reveal incorrect information. Finally, after having used the service being evaluated, the customer can tell whether the provided QoS fits the information service agents' predictions. The difference between what was expected and what was actually experienced is used to calculate the third component incentive $h_{k}(x)$. Of course, the latter can only be considered if the customer decides to have a transaction with the provider. In this case, $c$ will be set to 1 in the utility function, otherwise, $c$ will be equal to 0 .

It is important that each incentive has to be higher than the summation of the previous ones, that is to say $f_{k}(x)<\left|g_{k}(x)\right|$ and $f_{k}(x)+\left|g_{k}(x)\right|<\left|h_{k}(x)\right|$. It guarantees the incentive compatibility property which means that information service agents, as a utility maximizers, will reveal exactly what they believe about the service being evaluated or, in other words, they will tell the truth.

Lemma 1. The utility function $u_{k}(x)$ satisfies the following properties:

1. Revealing the true trust value about the service being evaluated is a Nash equilibrium strategy. 
2. If the service being evaluated is untrustworthy, revealing a false trust value is not a Nash equilibrium strategy.

3. If the provider is trustworthy, revealing a false trust value is a Nash equilibrium strategy.

Proof. As the first component is guaranteed, an information service agent has an incentive to lie only if the second incentive $g_{k}(x)$ is strictly positive and the third incentive is not obtained $(c=0)$. Therefore, the gained utility will be $\left|f_{k}(x)+g_{k}(x)\right|$. However, by truth telling, the information service agent can get the third incentive as well, so the gained utility will be $\left|f_{k}(x)+g_{k}(x)+h_{k}(x)\right|$. Consequently, telling the truth is the best strategy under Nash equilibrium. Adversely, if the service is untrustworthy, the agent will gain less by lying as $c .\left|h_{k}(x)\right|$ will be strictly negative. Thus, lying is not a Nash equilibrium. However, if the service is trustworthy but all the information service agents decide to lie, no one can gain a better payoff by deviating from the group's telling as $g_{k}(x)$ will be negative and $c .\left|h_{k}(x)\right|$ null, which proves the third property.

The problem we would like to investigate is the situation where a web service, aiming to join a community, pays the information service agent each time he gets chosen to join a community after being referenced by this agent. The web service could be tempted to cheat and pay more the information service agents so that they improve his reputation when informing a community master. If the reward a web service gives to information service agents to fake their opinion is big enough, these agents can be tempted to lie. In that case, a community will hire the web service and maybe expect more than what the service can really handle.

\subsection{The Modelled Game}

In the set up game, there is a number of involving agents: (1) a typical community $\left(M_{i}\right)$; (2) a typical single web service $\left(S_{j}\right)$; and (3) a typical information service agent $\left(I_{k}\right)$. In the set up game, both the community of web services and single web service handle user requests according to their capacities. In the ideal case, $M_{i}$ and $S_{j}$ desire to be neither overloaded, nor idle. When $M_{i}$ asks $I_{k}$ for information about the quality of $S_{j}$, a reputation value is produced. This value, representing what $I_{k}$ reports to be the reputation of $S_{j}$, is assigned to the triplet $\left(M_{i}, S_{j}, I_{k}\right)$. Such a value is saved by $M_{i}$ in registries to keep a record of who reported what about the different services so the value of the actual quality can be compared with the provided one. We define strategies of truth telling and lying within strategy profile $s t=\{0,1\}$, where 0 and 1 respectively reflect lying and telling the truth. As rational agent, an information service agent would choose his strategy based on the gained utility. For instance, he might obtain an acceptable offer that encourages him to lie and provide inaccurate information. Meanwhile, there might be other effective parameters that encourage agents to provide truthful information. This paper mainly focuses on the truth telling issues and discusses the way of converging towards this situation.

\subsection{Payments}

The payments information service agents receive can come from $M_{i}$ and from the service being evaluated. For simplicity reasons and to avoid notation confusion, we use 
three simple variables $\alpha, \beta$, and $\gamma$ that refer to the previously described incentives $f_{k}(x)$, $g_{k}(x)$, and $h_{k}(x)$ in Equation $11 M_{i}$ pays $\alpha$ to $I_{k}$ as an incentive to provide information about $S_{j}$. Parameter $\beta$ is the payment $M_{i}$ gives to $I_{k}$ after collecting reports about $S_{j}$ from $I_{0}, \ldots, I_{n} . \beta$ is calculated in Equation 2 where $\operatorname{Tr}_{M_{i}}^{I_{x}}$ represents the value of trust (confidence) $M_{i}$ has towards $I_{x}$, and $R r_{I_{x}}^{S_{j}}$ represents the value of the reputation of $S_{j}$ reported by $I_{x} . \beta$ is then a decreasing logarithmic function $(0<b<1)$ on the difference between the average reported value by all the information service agents and the value reported by $I_{k}$. Consequently, $I_{k}$ receives the highest payment for the closest reported value to this average (we assume that this distance is always different from 0 , otherwise a fixed payment, close to the highest, can be given).

$$
\beta=\log _{b}\left(\left|\frac{\sum_{x=0}^{n} \operatorname{Tr}_{M_{i}}^{I_{x}} R r_{I_{x}}^{S_{j}}}{\sum_{x=0}^{n} \operatorname{Tr}_{M_{i}}^{I_{x}}}-\operatorname{Rr}_{I_{k}}^{S_{j}}\right|\right)
$$

$\gamma$ is the payment $M_{i}$ gives to $I_{k}$ if $M_{i}$ has registered $S_{j}$ in $C_{i}$ and evaluated his reputation. After this evaluation, $M_{i}$ can compare $R r_{I_{x}}^{S_{j}}$ to $R o_{M_{i}}^{S_{j}}$ and pay $I_{k}$ with a value of $\gamma$, which is computed in Equation 3 , where $R r_{I_{x}}^{S_{j}}$ represents the value of the reputation of $S_{j}$ reported by $I_{x}$, and $R o_{M_{i}}^{S_{j}}$ represents the value of the actual reputation of $S_{j}$ observed by $M_{i}$. As in Equation 2 , $I_{k}$ receives the highest payment when the reported reputation value is the closest to the observed one. If the distance between these two values is 0 , a fixed payment can be set.

$$
\gamma=\log _{b}\left(\left|\operatorname{Rr}_{I_{x}}^{S_{j}}-\operatorname{Ro}_{M_{i}}^{S_{j}}\right|\right)
$$

The single web service $S_{j}$ can pay $\pi$ to $I_{k}$ in order to encourage him to increase his reputation when reporting to communities. This payment will only be received if $M_{i}$ chooses to add $S_{j}$ to the community. As shown in Equation 4 this payment is an exponentially decreasing function on the actual web service's reputation $R r^{S_{j}}$, which means if $S_{j}$ has a high reputation, only a small payment can be given to $I_{k}$, but if this reputation is low, $S_{j}$ has to reward high $I_{k}$ if he is getting selected by $M_{i}$. In this equation, $\lambda$ is application-dependent that measures the decreasing slope. In this paper, we assume $\lambda=1$.

$$
\pi=e^{-\lambda R r^{S_{j}}}
$$

\section{Cases Overview}

In this section, we analyze different cases using a game involving two players $(I . S$ for a typical information service agent and O.I.S for the other information service agents). Each game is represented as a table where the rows show the strategies of I.S and the columns indicate the strategies of O.I.S. Each cell of the table represents the action profile, i.e. the outcome that each player has according to the adopted strategy. The first outcome is for I.S and the second one for O.I.S. If the received payment is negative, we use the superscript - . For example, $\beta^{-}$means a negative $\beta$, otherwise, $\beta$ is positive.

We focus on the cases where $S_{j}$ is honest (with good and bad QoS). Due to space limitations, we skip the case where $S_{j}$ is dishonest, however, this case could be easily 
generalized. In our considered cases, $S_{j}$ does not try to corrupt I.S and O.I.S by rewarding them. Therefore, we will only take into account the first three payments $\alpha, \beta$ and $\gamma$. Then, we apply the same analysis on the dishonest $S_{j}$, where the information service agents can receive the incentive $\pi$ in order to improve fraudulently the information they report about $S_{j}$.

$\boldsymbol{S}_{\boldsymbol{j}}$ has good QoS. The assigned payoff regarding each strategy is set up in Table 1. If every information service agent tells the truth, i.e. informing $M_{i}$ that $S_{j}$ is good, everyone will receive a maximum payment of $\alpha+\beta+\gamma$. Indeed, the information services will receive $\alpha$ in reward for processing the request. They will gain $\beta$ because the value of the report that each information service agent will give will be close to the average of all the reported values. Finally, they will receive the third payment $\gamma$ since the observed reputation and announced one will be close to each other.

Table 1. Honest single web services - $S_{j}$ has good QoS

\begin{tabular}{|c|c|c|}
\hline & \multicolumn{2}{|l|}{ O.I.S } \\
\hline & Truth & Lie \\
\hline I.STruth & $(\alpha+\beta+\gamma),(\alpha+\beta+\gamma)$ & $\left(\alpha+\beta^{-}\right),\left(\alpha+\beta_{-}\right)$ \\
\hline Lie & $\left(\alpha+\beta^{-}+\gamma^{-}\right),\left(\alpha+\beta_{-}+\gamma\right)$ & $(\alpha+\beta),(\alpha+\beta)$ \\
\hline
\end{tabular}

If $I . S$ decides to lie while O.I.S continue to tell the truth, I.S will degrade his total payment to $\alpha+\beta^{-}+\gamma^{-}$. He will still receive $\alpha$ but a negative $\beta$ and $\gamma$ as computed in Equations 2 and 3 because the reputation he reported will be far from the average and observed reputation. On the other hand, O.I.S will also perceive a degradation in their total payment. In fact, because one information service agent decided to report a reputation totally different from those reported by the others, the average will be smaller than if every information service agent had reported close values. In this case we use $\beta_{-}$instead of $\beta$. If O.I.S decide to change their strategies and lie, they will only get $\alpha$ and $\beta_{-}$as payment. Majority announcement that $S_{j}$ is bad implies that the community will not accept him, and therefore the third payment $\gamma$ will not be granted. As $I . S$ did not change his strategy, he will get $\alpha$ and a negative $\beta$ because of the big distance from the average and not $\gamma$ because $S_{j}$ will not enter the community. If everyone decides to lie, all the information services will get $\alpha$ and $\beta$ and nobody will receive $\gamma$ as the service will not join the community. Thus, there is an incentive to tell the truth for everyone because it corresponds to the situation that guarantees the maximum payment $\alpha+\beta+\gamma$. This game has the following properties:

Lemma 2. Telling the truth by all the information service agents is the only Pareto optimal and Nash equilibrium (Pareto-Optimal-Nash).

Proof. The proof is straightforward from Table 1 since any change of strategy of telling the truth would degrade other agents' payoffs and no other situation has this property.

Lemma 3. Lying by all the information service agents is Pareto optimal.

Proof. The proof is straightforward from Table 1 since any change of one's strategy of lying would degrade the opponent's payoff. 
$\boldsymbol{S}_{\boldsymbol{j}}$ has bad QoS. In this second case, we assume that the honest $S_{j}$ provides a bad QoS.

Table 2. Honest single web services - $S_{j}$ has bad QoS

O.I.S.

\begin{tabular}{|c|c|c|}
\cline { 2 - 3 } \multicolumn{1}{c|}{} & Truth & Lie \\
\cline { 2 - 3 } I.S. Truth & $(\alpha+\beta),(\alpha+\beta)$ & $\left(\alpha+\beta^{-}+\gamma\right),\left(\alpha+\beta_{-}+\gamma^{-}\right)$ \\
\hline Lie & $\left(\alpha+\beta^{-}\right),\left(\alpha+\beta_{-}\right)$ & $\left(\alpha+\beta+\gamma^{-}\right),\left(\alpha+\beta+\gamma^{-}\right)$ \\
\hline
\end{tabular}

If every player tends to tell the truth and reveals that $S_{j}$ has bad QoS, they will all get a payment of $\alpha+\beta$ (see Table 2). If $I . S$ decides to modify his strategy and lie by announcing that $S_{j}$ has good QoS, he will degrade his total payment. He will still receive $\alpha$ but a negative $\beta$ because the reputation he announced is far from the average. However, O.I.S will get the two first payments but the second one will be slightly decreased in comparison to the previous situation. Therefore, they will perceive $\alpha+\beta_{-}$. In the next situation, assume that O.I.S change their strategies and start to lie. Because the majority of information service agents will declare $S_{j}$ as good, the web service will join the community. Soon, $M_{i}$ will realize that $S_{j}$ has actually bad QoS. Therefore, the group of O.I.S will receive $\alpha+\beta_{-}+\gamma^{-}$. On the other hand, $I . S$ who kept his strategy of telling the truth will get $\alpha+\beta^{-}+\gamma$. Indeed, the information service will receive a negative $\beta$ but will be rewarded by $\gamma$ as he reported correctly that $S_{j}$ has bad QoS. If everyone decides to lie, all the information service agents will get $\alpha+\beta+\gamma^{-} \cdot \gamma$ will be negative because $S_{j}$ will join the community, so $M_{i}$ will discover his bad QoS. The following property results directly from Table 2 .

Lemma 4. Telling the truth is the only Pareto-Optimal-Nash.

\section{Related Work}

In many frameworks proposed in the literature, service selection and task allocation are regulated based on the reputation parameter [10]11]. In [1], the proposed framework regulates the service selection based on the trust policies expressed by the service users. In [9], authors propose ontology for quality of service. Users compute the web services' reputation using ratings. The frameworks proposed in [3]8] address effective reputation mechanism for web services. All these models address the reputation in environments where web services function alone. In such models, web service efficiency is not discussed in details and in general, balancing the market share with the capacity is not considered as an issue for web service besides his reputation.

There have been few work addressing the communities of services. The objective is to facilitate and improve the process of service selection and effectively regulate the process of request and task allocation [2]. In [4], authors propose a reputation-based architecture for communities and investigate the collusion scenarios that might falsely increase communities' reputation in the network. In [5], the authors mainly address the overall assessed reputation that is used as a main reason for service selection. The authors do not consider truth/lie telling analysis as a factor impacting service selection. 


\section{Conclusion}

The contribution of this paper is the proposition of a game-theoretic based model to analyze the best efficiency characteristics for the active services in open networks. The proposed framework considers the chances of web services in joining a community in different cases with truthful and lying information service agents. The proposed game analyzes the existing Nash equilibrium and situations where the maximum payoff is obtained. Our model has the advantage of being simple and taking into account three important factors: (1) rational services seek better status in the environment by joining the community; (2) rational information service agents obtain higher payoff by truth telling; and (3) the community is obtaining more effective web services while the information service agents challenge for providing truthful information. As future work, we plan to consider the user role in the game to obtain more accurate results when users act rationally. Moreover, we would like to achieve a collusion resistant efficiency mechanism, which is still an open problem in open environments.

\section{References}

1. Ali, A.S., Ludwig, S.A., Rana, O.F.: A cognitive trust-based approach for Web service discovery and selection. In: Proc. of the Euro. Conference on Web Services, pp. 38-40 (2005)

2. Jacyno, M., Bullock, S., Luck, M., Payne, T.R.: Emergent Service Provisioning and Demand Estimation through Self-Organizing Agent Communities. In: 8th International Joint Conference on Autonomous Agents and Multiagent Systems, pp. 481-488 (2009)

3. Kalepu, S., Krishnaswamy, S., Loke, S.W.: A QoS metric for selecting Web services and providers. In: Proc. of the 4th International Conference on Web Information Systems Engineering Workshops, pp. 131-139 (2003)

4. Khosravifar, B., Bentahar, J., Moazin, A., Thiran, P.: Analyzing communities of web services using incentives. International Journal of Web Services Research 7(3), 30-51 (2010)

5. Khosravifar, B., Bentahar, J., Thiran, P., Moazin, A., Guiot, A.: An approach to incentivebased reputation for communities of Web services. In: Proc. of IEEE 7th International Conference on Web Services, pp. 303-310 (2009)

6. Khosravifar, B., Bentahar, J., Moazin, A., Maamar, Z., Thiran, P.: Analyzing communities vs. single agent-based web services: trust perspectives. In: Proc. of the IEEE international Conference on Services Computing, pp. 194-201 (2010)

7. Maamar, Z., Subramanian, S., Thiran, P., Benslimane, D., Bentahar, J.: An approach to engineer communities of web services: Concepts, architecture, operation, and deployment. International Journal of E-Business Research 5(4), 1-21 (2009)

8. Maximilien, E.M., Singh, M.P.: Conceptual model of Web service reputation. SIGMOD Record 31(4), 36-41 (2002)

9. Maximilien, E.M.: Multiagent system for dynamic web services selection. In: The 1st Workshop on Service-Oriented Computing and Agent-based Eng., pp. 25-29 (2005)

10. Rosario, S., Benveniste, A., Haar, S., Jard, C.: Probabilistic QoS and soft contracts for transaction based web services. In: IEEE International Conference on Web Services, pp. 126-133 (2007)

11. Ruth, M., Shengru, T.: Concurrency issues in automating RTS for Web services. In: IEEE International Conference on Web Services, ICWS 2007, pp. 1142-1143 (2007) 